\title{
Proliferative effect of high glucose is modulated by antisense oligonucleotides against fibronectin in rat endothelial cells
}

\author{
S. R oy ${ }^{1}, T$. R oth ${ }^{2}$ \\ ${ }^{1}$ Schepens Eye Research Institute and Department of Ophthalmology, Harvard Medical School, Boston, Massachusetts, USA \\ ${ }^{2}$ Division of Plastic Surgery, University of Massachusetts Medical Center, Worcester, Massachusetts, USA
}

Summary Increased synthesis of fibronectin is associated with the development of basement membrane thickening - a characteristic lesion of diabetic microangiopathy, and it may affect the function of vascular cells. Because antisense technology offers the possibility to modulate specific gene expression, we investigated the effect of antisense phosphorothioate oligonucleotides directed against fibronectin mRNA on fibronectin synthesis and cell proliferation in a cell line derived from rat microvascular endothelium and cultured under high $(30 \mathrm{mmol} / \mathrm{l})$ glucose conditions. The rat endothelial cells grown in high glucose medium for $5.6 \pm 1.3$ days exhibited increased cell proliferation compared to control cells grown in $5 \mathrm{mmol} / \mathrm{l}$ glucose $(149 \%$ of control, $p=0.006)$. Fibronectin protein and mRNA levels (determined by Western blotting and reverse transcription-polymerase chain reaction) were also increased to $157 \%$, $p=0.012$ and $178 \%, p=0.034$ of control, respectively. However, when cells grown in high glucose medium were transfected with $0.4 \mathrm{mmol} / \mathrm{l}$ fibronectin-antisense phosphorothioate oligonucleotides in the presence of cationic liposomes, the cell number, fibronectin protein, and mRNA levels decreased compared to untransfected cells grown in high glucose medium to 102,69 , and $107 \%$ of control, respectively. This study shows that fibronectin antisense oligonucleotides targeted to the translation initiation site of the fibronectin transcript specifically reduce fibronectin synthesis in rat endothelial cells and the proliferative effect of high glucose concentrations. [Diabetologia (1997) 40: 1011-1017]

Keywords Basement membranes, fibronectin, gene expression, high glucose, antisense oligonucleotides.
Fibronectin is a large glycoprotein identified as one of the principal components of the extracellular matrix [1]. Several studies indicate that increased synthesis of fibronectin by vascular endothelial cells may play a key role in the initiation and progression of thickened basement membrane, a characteristic lesion of diabetic microangiopathy [2-4]. Excess

Received: 16 January 1997 and in final revised form: 22 April 1997

Corresponding author: Dr. S. Roy, Schepens Eye Research Institute, Harvard Medical School, 20 Staniford Street, Boston, MA 02114, USA

A bbreviations: RT-PCR, Reverse transcription-polymerase chain reaction; PS oligos, antisense phosphorothioate oligonucleotides, GCG, genetic computer group. fibronectin has been shown to alter cell-cell interactions [4] and cell-substratum interactions [5] with consequences for cell spreading, migration, and proliferation [4]. We have previously reported that cultured human endothelial cells grown in high glucose medium, and tissues from experimental diabetic rats, exhibit increased expression of fibronectin $[6,7]$. Recently we have reported increased synthesis of fibronectin in retinal microvessels of human diabetic patients [2], and in retinas of galactosaemic rats that develop diabetic-like retinopathy [8]. Since antisense oligonucleotides offer a potential means of inhibiting expression of individual genes [9, 10], we hypothesized that by reducing fibronectin synthesis using antisense phosphorothioate modified oligonucleotides (PS oligos), the proliferative effects of high glucose may be modulated. Unmodified oligonucleotides 
have limited effect, and are rapidly degraded, therefore, we employed antisense oligonucleotides with phosphorothioate modification that impart stability to the oligonucleotides, and retard degradation by endonucleases. The modification constitutes the replacement of one oxygen of each internucleotide linkage with a sulphur [9].

Antisense activity is dependent upon several criteria, including selection of specific target sequence and vehicles for cellular uptake of the oligonucleotides. Although several target sites including 5'cap region [11], splice donor-acceptor site [12], and translation initiation site $[11,13]$ have been successfully utilized to modulate specific gene expression, no consensus exists as to which target site is most effective. In this study we have selected oligonucleotides complementary to 17 nucleotides of the fibronectin sequence [14] encompassing the AUG translation initiation codon since antisense oliogonucleotides targeted against a similar site were most effective in inhibiting intercellular adhesion molecules (ICAM-1) in cultured endothelial cells [13]. As a vehicle for oligonucleotide transfection, we used Lipofectin, a liposome formulation, because of its high efficiency in transfecting oligonucleotides into eukaryotic cells [15].

\section{Materials and methods}

Cells and reagents. A rat endothelial cell line derived from microvascular endothelium of epididymal fat pads and ascertained positive for von Willebrand protein by immunofluorescence microscopy was used in this study [16]. Cells were maintained in M199 (Life Technologies, Gaithersburg, Md., USA) containing $10 \%$ fetal bovine serum (Sigma, St. Louis, Mo., USA), antibiotics, and supplemented with normal $(5 \mathrm{mmol} / \mathrm{l})$ or high $(30 \mathrm{mmol} / \mathrm{l}) \mathrm{D}$-glucose. Lipofectin, a cationic formulation consisting of dioleoyl phosphatidylethanolamine (DOPE) and N-[1-(2,3-dioleyloxy) propyl]-N,N,N-trimethylammonium chloride (DOTMA) commercially available as Lipofectin (Life Technologies) were used as carriers for transfection of the PS oligos into cultured cells. At the semi-confluent stage, cells were washed twice with Opti-MEM (Gibco, Grand Island, N. Y., USA) pre-warmed at $37^{\circ} \mathrm{C}$, and transfected with PS oligos at a concentration of $0.4 \mu \mathrm{mol} / 1$ for $4 \mathrm{~h}$ at $37^{\circ} \mathrm{C}$. At the end of the $4 \mathrm{~h}$ period, Opti-MEM containing PS oligos was replaced with M199 and allowed to incubate for $48 \mathrm{~h}$ at $37^{\circ} \mathrm{C}$.

Cell count. Endothelial cells plated in six-well dishes and grown in normal medium or high glucose medium were transfected with fibronectin-antisense or fibronectin-sense PS oligos. At semi-confluency cells were washed three times with Ca-free phosphate buffered saline (PBS), trypsinized and counted in a Coulter Counter in duplicate for each experiment.

Oligonucleotides. The sequence for the 17-mer antisense fibronectin oligonucleotides 5'-CCT GAG CAT CTT GAG TG $3^{\prime}$, used in this study, was selected from a published sequence of the rat fibronectin mRNA (accession number X15906 via GCG) [14]. A 17-mer sense sequence similar to the translation initiation region was used as control. The antisense and sense oligonucleotides were prepared using amidite chemistry with phosphorothioate modification, and obtained commercially (Oligos Etc., Willsonville, Ore., USA).

R everse transcription-polymerase chain reaction and Southern analysis. In each experiment, RNA from cells grown in normal medium or high glucose medium were processed in parallel with the RNA from cells transfected with fibronectin-PS oligos and grown in normal or high glucose medium. Reverse transcription was performed [8] with random hexamers followed by PCR with fibronectin and actin primers [8] and cycle conditions as previously reported [8]. PCR was designed to measure the level of fibronectin expression relative to the expression of an endogenous internal standard gene ( $\beta$-actin). Actin mRNA level was used as an internal control because it has been previously shown not to be altered by either high glucose or the transfection process $[8,17]$. Fibronectin and $\beta$-actin cDNAs were generated in the same RT reaction and amplified in separate tubes containing 1 or $2 \mu \mathrm{l}$ volumes of the RT reaction because in pilot experiments densitometric values of PCR products generated from 1 and $2 \mu \mathrm{l}$ samples of reverse transcribed material showed amplification in the linear range for each cDNA.

Previous studies with retinal fibronectin mRNA level have indicated that 1 and $2 \mu \mathrm{l}$ samples of reverse transcribed material consistently represented amplification in the linear region (8). PCR products from control and experimental samples were resolved in the same gel, transferred onto nylon membrane, hybridized with fibronectin cDNA [14] for Southern blot analysis [18] and the densitometric values quantitated as described [19].

Immunofluorescence microscopy. To study the distribution pattern and relative amounts of fibronectin, immunofluorescence staining for fibronectin was performed on rat endothelial cells grown in normal medium or high glucose medium, and cells grown in high glucose medium transfected with fibronectin-antisense PS oligos. Briefly, cells grown to semi-confluency in two-chamber glass slides in medium M199 were transfected with antisense PS oligos, rinsed in PBS, fixed in $1 \%$ paraformaldehyde for $10 \mathrm{~min}$, washed in PBS, and treated with $2 \%$ bovine serum albumin (BSA) in PBS for 10 min to block nonspecific antibody binding. The cells were then incubated for $1 \mathrm{~h}$ at room temperature with polyclonal mouse anti-rat fibronectin antibody (Chemicon, Temecula, Calif., USA) diluted 1:500 in PBS containing 2\% BSA. After three PBS washes, the cells were exposed to $2 \% \mathrm{BSA}$ in PBS for $10 \mathrm{~min}$. Fluorescein isothiocyanate (FITC)-conjugated goat anti-mouse IgG (Sigma) was diluted 1:200 and incubated for $1 \mathrm{~h}$ in a moist chamber. After three PBS washes, coverslips were mounted in Slow-Fade (Molecular Probes, Eugene, Ore., USA). Negative control samples were processed in exactly the same way as the experimental groups except that the primary antibody was omitted. The cells were viewed and photographed using a Nikon UFX II photomicroscope equipped for epifluorescence illumination. Fibronectin-specific fluorescence was assessed by investigators without knowledge of the different experimental groups on a scale from 0 representing complete absence of fluorescence to 4 representing the highest intensity.

Immunoprecipitation. Rat microvascular endothelial cells from semi-confluent dishes were washed with PBS and the cellular proteins were extracted with lysis buffer containing $25 \mathrm{mmol} / \mathrm{l}$ Tris $\mathrm{pH}=7.5$ (Sigma), $1 \mathrm{mmol} / \mathrm{l}$ phenylmethylsulfonyl fluoride (Sigma), 5 mmol/l EDTA, and 0.1\% Triton X-100 (Sigma). The extracts were pre-adsorbed with pre-immune rat serum (Sigma) followed by protein G-Sepharose (1:3 slurry). Companion cultures were trypsinized and counted to determine cell number. The samples were equalized on the basis of 
cell number $\left(\sim 1.2 \times 10^{5}\right.$ cells $)$. Equal amounts of protein from cells grown in normal medium, high glucose medium, or high glucose medium transfected with antisense fibronectin PS oligos were incubated for $90 \mathrm{~min}$ at ambient room temperature with goat anti-rat fibronectin antiserum (Chemicon). The immune complexes were precipitated with protein G-Sepharose (Pierce, Rockford, Ill., USA). The immunoprecipitated fibronectin samples and molecular weight standards (BioRad, Melville, N. Y., USA) were resuspended and denatured in Laemmli buffer without reducing agents and electrophoresed on a $10 \%$ polyacrylamide gel containing $1 \%$ sodium dodecyl sulphate (SDS).

Transfer. Following electrophoresis, protein was transferred onto polyvinylidine difluoride (PVDF) membrane (BioRad) according to Towbin's procedure [20] using a semi-dry apparatus with a Towbin buffer system. Following transfer, the gel was stained with Coomassie blue to verify transfer of proteins to PVDF membrane. The molecular weight standard lane on the PVDF membrane was cut away, prewetted with methanol, and stained with amido black (BioRad). Presence of stain verified complete transfer of protein onto the membrane. The remaining portion of the membrane, with the separated proteins, was subjected to Western blot analysis.

Western blot. Western analysis was performed to examine the steady-state level of fibronectin expressed in these cells. The proteins transferred to the PVDF membrane were detected with the BioMax Chemiluminescent Detection System (Kodak, Rochester, N. Y., USA) and the goat anti-rat fibronectin antiserum. Briefly, the membrane was equilibrated with methanol, followed by PBS, and blocked with $5 \%$ nonfat milk. The blocking solution was removed and replaced with fresh blocking solution containing anti-fibronectin antiserum. The blot was washed with blocking buffer and then incubated with fresh blocking buffer containing donkey anti-goat IgG antibody conjugated with horseradish peroxidase enzyme (Jackson Laboratories, Inc., West Grove, Pa., USA). The membrane was washed as above, equilibrated in Chemiluminescent Detection System (CDS) buffer, and then applied to the Kodak chemiluminescence matrix. The membrane was exposed to XAR-5 Kodak film. Densitometric analysis of the luminescent signal was performed at non-saturating exposures with a laser scanning densitometer.

Statistical analysis. Data are expressed as mean \pm SD. Comparisons between groups were performed with analysis of variance (ANOVA), and Student's t-test. Statistical comparisons between the different groups in the immunofluorescent studies were performed by the Kruskal-Wallis test. A level of $p$ less than 0.05 was considered as statistically significant.

\section{Results}

Down regulation of fibronectin expression in transfected cells. We determined that the fibronectin-antisense PS oligos specifically reduce fibronectin expression in cells grown in normal medium or high glucose medium (Fig.1). Cells grown in high glucose medium exhibited an increased fibronectin mRNA level ( $178 \%$ of control, $p=0.034)$, and was significantly reduced ( $p=0.015)$ by antisense PS oligos to near normal level (107\% of control), without affecting the actin mRNA levels. Similarly, cells grown in normal

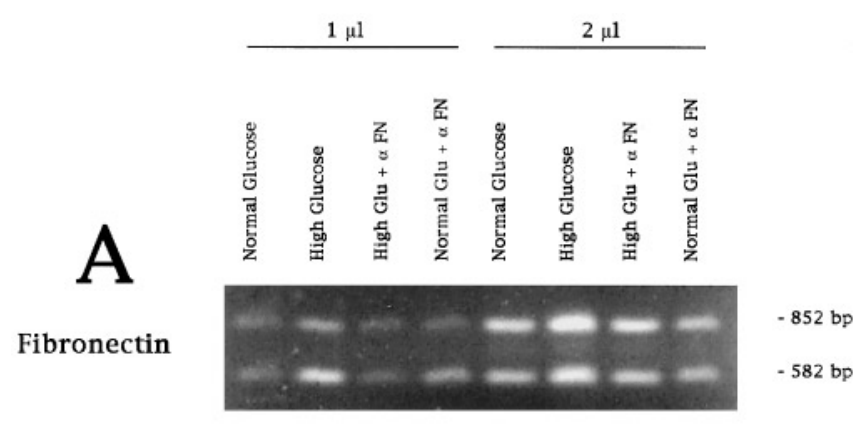

$\beta-$ Actin

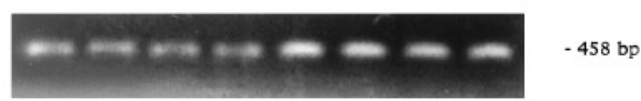

B

Fibronectin

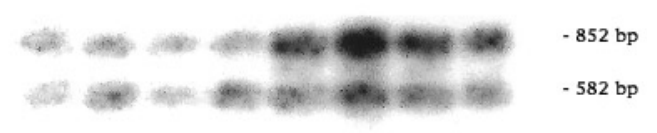

$\beta$ - Actin

$-458 \mathrm{bp}$

Fig. 1A , B. Effect of fibronectin-antisense oligonucleotides on mRNA level of fibronectin in rat endothelial cells. (A) PCR amplification products for fibronectin and $\beta$-actin as detected on ethidium bromide-stained agarose gel with 1 or $2 \mu l$ samples of reverse-transcribed cDNA. PCR with fibronectin primer pair encompassing alternatively spliced EIIIA exon produces two bands: $582 \mathrm{bp}$ and $852 \mathrm{bp}$; PCR with actin primer pair generates a single $458 \mathrm{bp}$ band. (B) Corresponding Southern blot analysis

medium transfected with fibronectin antisense oligonucleotides exhibited decreased fibronectin mRNA level (77\% of control). The actin mRNA levels were very similar in the three groups: $98 \%$ of control in cells grown in high glucose medium, $95 \%$ of control in cells grown in high glucose medium transfected with antisense oligos, and $105 \%$ of control in cells grown in normal medium transfected with antisense oligos (Fig.1). In each experiment, fibronectin mRNA level in cells exposed to sense oligos was not altered (98\% of control). The densitometric readings of signals of PCR-amplified products for fibronectin and $\beta$-actin as detected on ethidium bromide-stained agarose gel (Fig. 1A) and those of the corresponding Southern blot (Fig. 1B) showed excellent correlation $(r=0.96)$. Cells grown in normal medium and transfected with antisense oligos were studied to compare whether the extent of fibronectin down regulation by antisense oligos was any different in cells grown in high glucose medium compared to cells grown in normal medium, and whether the titred concentration of antisense PS oligos was within a range that was not lethal to the cells (Fig. 2). Interestingly, cells grown in high glucose medium and transfected with fibronectin-antisense PS oligos exhibited a significant 


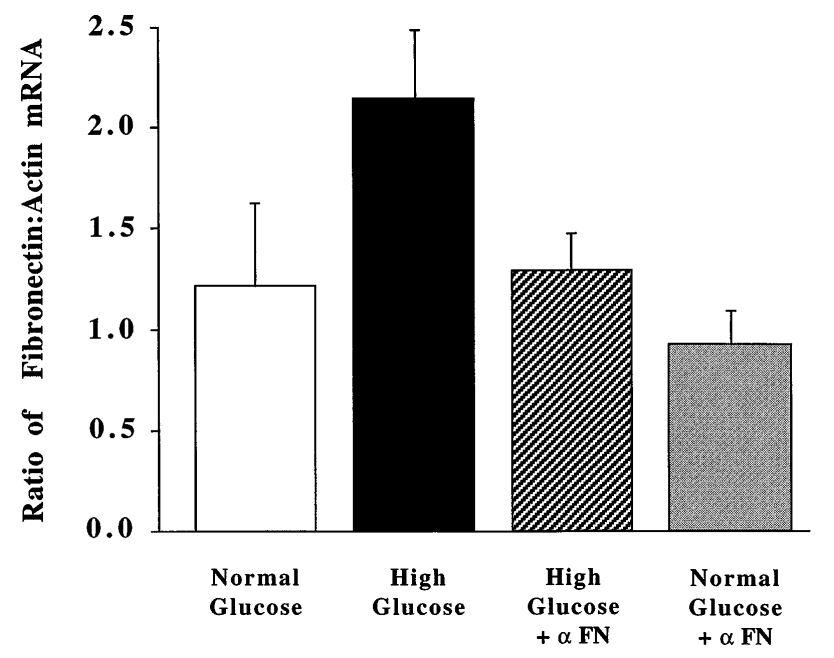

Fig. 2. Selective reduction of fibronectin mRNA level by antisense oligonucleotides. Ratio of fibronectin:actin mRNA levels in cells grown in normal medium $\square$, high glucose medium $\square$, high glucose medium transfected with fibronectin-antisense oligonucleotides $\mathbb{Z}$, and normal medium transfected with fibronectin-antisense oligonucleotides [1. Increased fibronectin:actin mRNA ratio in cells grown in high glucose medium $(p<0.04)$ compared to cells grown in normal medium. Reduced fibronectin:actin mRNA ratio in cells grown in high glucose medium transfected with fibronectin-antisense oligonucleotides compared to cells grown in high glucose medi$\operatorname{um}(\mathrm{p}<0.02),(\mathrm{n}=3)$

reduction in fibronectin mRNA level (from 178 to $107 \%$ of control, $p=0.01)$. However, when cells grown in normal medium are transfected with PS antisense oligos, the downregulation of fibronectin mRNA level is small (from 100 to $77 \%$ of control) $(p=0.4)$ (Fig. 2$)$. This finding that fibronectin-antisense PS oligos down regulates fibronectin mRNA level more effectively in cells grown in high glucose medium may have important implications regarding its mode of action.

I mmunofluorescence microscopy. Using immunofluorescence microscopy we determined the distribution and relative quantity of fibronectin in rat endothelial cells. Fibronectin was detected in the cells from control and treated groups. In cells grown in high glucose medium the fluorescence pattern suggested presence of fibronectin as an organized scaffold on the cells (Fig.3). The fibronectin immunofluorescence was more intense in the cells grown in high glucose medium; however, the intensity of immunofluoresence

Fig. 3A-C. Fibronectin immunoreactivity in rat endothelial cells. Representative photomicrographs of $(\mathbf{A})$ cells grown in normal glucose medium, (B) cells grown in high glucose medium, and (C) cells grown in high glucose medium transfected with fibronectin-antisense oligonucleotides. Magnification $\times 580$
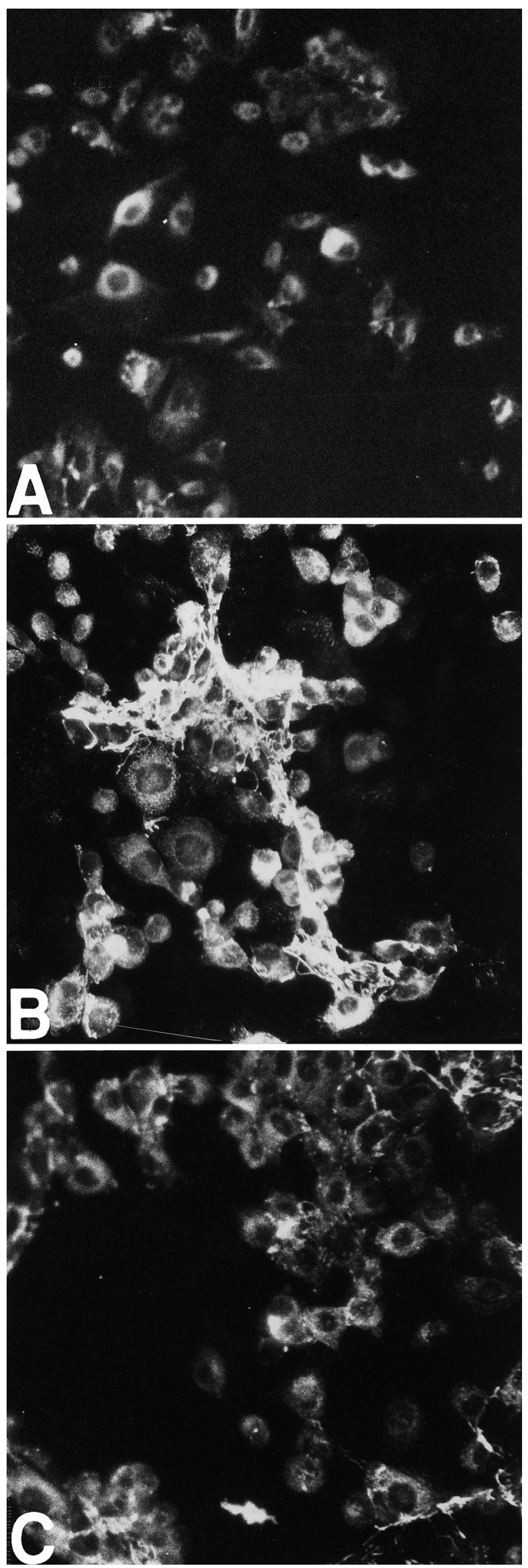


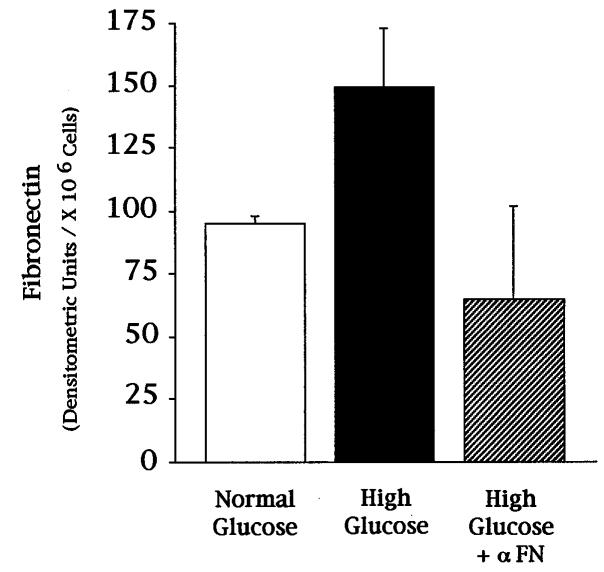

Fig. 4. Effect of fibronectin-antisense oligonucleotides on fibronectin protein level. Relative amount of fibronectin protein in cells grown in normal medium $\square$, high glucose medium $\square$, and high glucose medium transfected with fibronectin-antisense oligonucleotides $\mathbb{Z}$. In cells grown in high glucose medium fibronectin was significantly increased $(p<0.02)$ compared to cells grown in normal medium, and significantly reduced in cells transfected with fibronectin-antisense oligonucleotides $(p<0.03)$

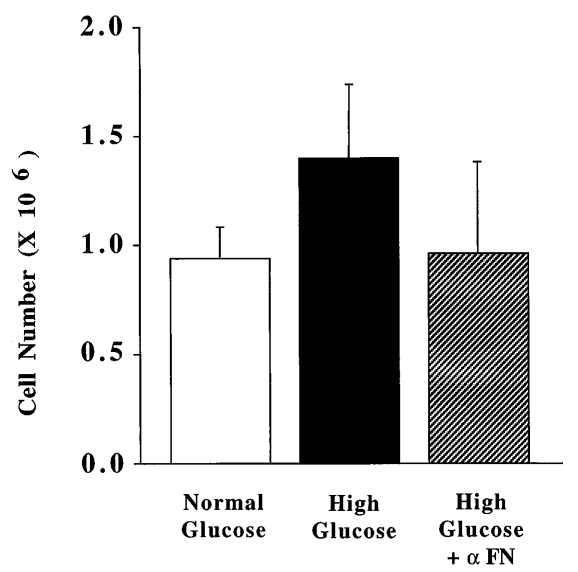

Fig. 5. Effect of fibronectin-antisense oligonucleotides on proliferation of rat endothelial cells. Cell number from cells grown in normal glucose medium $\square$, high glucose medium $\square$, and high glucose medium transfected with fibronectin-antisense oligonucleotides $\mathbb{Z}$. Cells grown in high glucose medium exhibit increased cell number $(p<0.01)$ compared to cells grown in normal medium. Cells transfected with fibronectin-antisense oligonucleotides exhibit significant reduction in cell number compared to cells grown in high glucose medium $(p<0.05)$

decreased when the cells were transfected with fibronectin-antisense PS oligos. The semiquantitative analysis of fibronectin from immunofluorescence yielded a score of $0.8 \pm 0.2$ for cells in normal medium, $2.5 \pm 0.5$ for cells in high glucose medium, and $1.3 \pm 0.4$ for cells transfected with fibronectin-antisense PS oligos, a significant reduction $(p=0.01)$ (Fig. 3$)$.

Western analysis of fibronectin level. The relative amount of fibronectin protein was determined in the cell extract of three groups of cells: cells grown in normal medium, high glucose medium, and cells grown in high glucose medium transfected with fibronectin-antisense PS oligos. Results from four experiments indicated that cells grown in high glucose medium exhibited increased fibronectin ( $157 \%$ of control, $p=0.012$ ), that was significantly reduced when transfected with antisense oligos (69\% of control, $p=0.027)$ (Fig. 4$)$.

Cell proliferation is modulated by antisense oligonucleotide targeted to fibronectin. In seven experiments, rat endothelial cell proliferation in high glucose was compared to those grown in normal medium. Cells grown in high glucose medium for $5.6 \pm 1.3$ days exhibited increased cell numbers compared to cells grown in normal medium $\left(1.4 \pm 0.34 \times 10^{6}\right.$ vs $0.94 \pm 0.18 \times 10^{6}, p=0.006$,) (Fig. 5). However, when cells grown in high glucose medium were transfected with $0.4 \mu \mathrm{mol} / 1$ antisense PS oligos, the cell number decreased significantly to near normal level: cells grown in high glucose medium $1.4 \pm 0.34 \times 10^{6}$ vs high glucose medium transfected with fibronectin-antisense oligos $0.96 \pm 0.42 \times 10^{6}, p=0.04$ ) (Fig. 5). In four cell proliferation experiments in which cells were grown for a fixed duration of 5 days, antisense fibronectin oligos exhibited an inhibitory effect on cell proliferation: cells grown in high glucose medium exhibited increased proliferation compared to cells grown in normal medium $\left(1.39 \pm 0.21 \times 10^{6}\right.$ vs $\left.0.83 \pm 0.15 \times 10^{6}, p=0.018\right)$ and the cell number was significantly reduced to $\left(0.9 \pm 0.17 \times 10^{6}, p=0.035\right)$ when transfected with fibronectin antisense oligonucleotides. The results indicate that irrespective of whether the cells were grown for a fixed 5-day duration or 4-6 days, the proliferative behaviour of these cells was reduced by the antisense oligonucleotides directed at the fibronectin transcript. Control experiments were performed to study the effects of lipofectin, the carrier molecule. Cells grown in normal medium, compared to cells grown in normal medium in the presence of lipofectin showed no difference ( $98 \%$ of control) indicating that the carrier had no effect on rat endothelial cell proliferation (Fig.6). As control, a second group of experiments were performed to determine whether the reduction in cell number by fibronectin-antisense PS oligos was due to the specificity of the antisense sequence. When cells grown in high glucose medium were transfected with sense-fibronectin PS oligos and compared to cells grown in high glucose medium, no difference in cell number was observed ( $94 \%$ of control) (Fig. 6).

\section{Discussion}

As in earlier studies with human endothelial cells [6, 18], we observed high glucose-induced fibronectin overexpression in rat microvascular endothelial cells. 


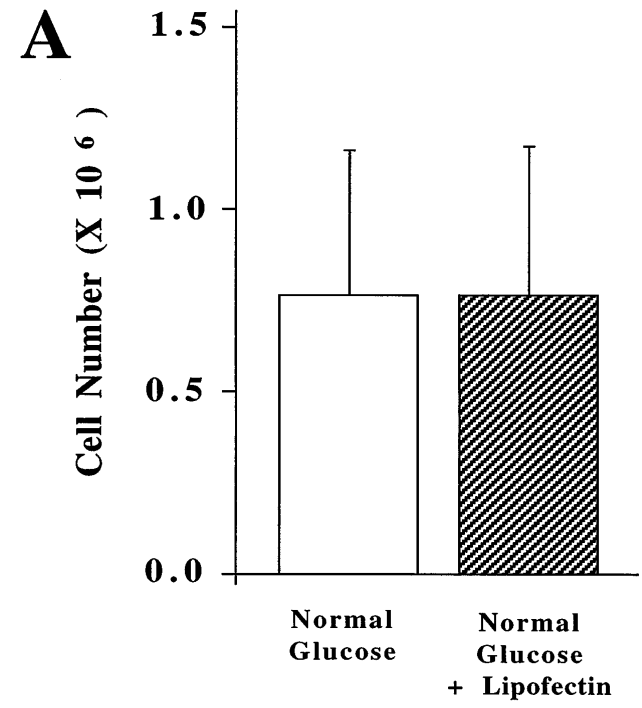

Fig. 6A , B . Effect of lipofectin or sense-fibronectin oligonucleotides in rat endothelial cells. (A ) cells grown in normal medium $\square$, compared to cells grown in normal medium in the presence of lipofectin $\mathbb{Z}$; (B) cells grown in high glucose medium $\square$, compared to cells grown in high glucose medium transfected with fibronectin-sense oligonucleotides $\mathbb{Z}$

This study indicates specific reduction of excess fibronectin synthesis by antisense phosphorothioate oligonucleotides targeted against fibronectin mRNA results in decreased cell proliferation. Although translational arrest may contribute to the down regulation of fibronectin synthesis, reduced levels of fibronectin mRNA observed in RT-PCR data suggests RNAse $\mathrm{H}$ activity and/or transcriptional arrest play a major role in down regulation of fibronectin synthesis.

As mentioned earlier, the choice of an optimal target for antisense application includes several sites $-5^{\prime}$ cap [21], AUG translation initiation codon [22], intron-exon boundary [12], and the 3'-untranslated region [13] - all of which have been successfully applied by different investigators with varying levels of efficiency. In this study we determined that the area encompassing the translation initiation codon of fibronectin transcript is an effective target site for down regulation of fibronectin synthesis by fibronectin-antisense PS oligos. Interestingly, the fibronectin-antisense PS oligos not only reduced fibronectin mRNA levels as anticipated, but that the reduction was selectively greater in cells grown in high glucose medium than in cells grown in normal medium. Although it is difficult to explain this phenomenon, a possible explanation is that the presence of excess fibronectin transcripts in cells grown in high glucose medium may provide more targets and increase the likelihood for fibronectin-antisense oligonucleotides to bind to its target sequence. When fibronectin mRNA is bound to fibronectin-antisense PS oligos, an RNA-DNA hybrid

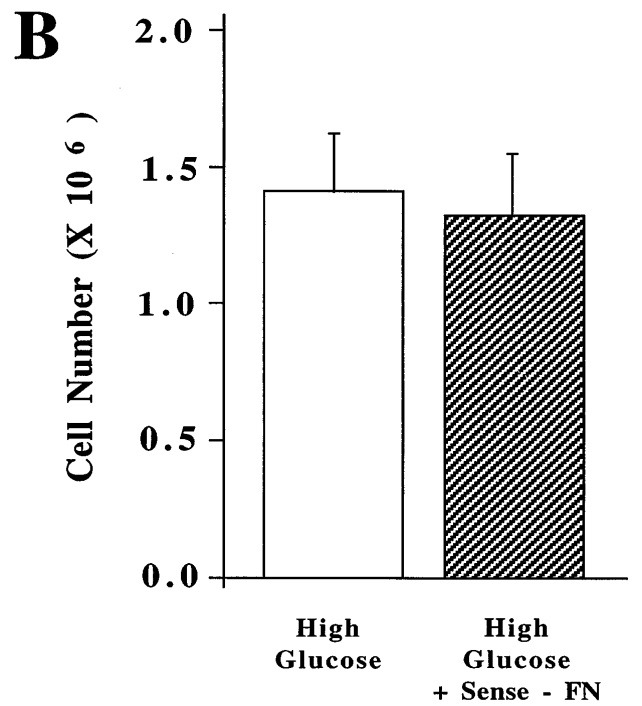

molecule is formed. Such a duplex structure would inhibit the translational process or lead to the destruction of fibronectin mRNA by RNaseH activity, and result in reduction of overall fibronectin synthesis.

In this study one of our goals was to test whether the selected sequence for the fibronectin-antisense oligonucleotides was specific and effective in down regulating fibronectin synthesis in rat endothelial cells because these antisense oligonucleotides may be useful in attempting down regulation of fibronectin expression in animal models of diabetic retinopathy such as the galactosaemic rat that exhibits increased fibronectin mRNA level [8]. Since these cells grown in high glucose medium exhibit increased fibronectin synthesis they offered an adequate model to test the antisense effect of the PS oligos on fibronectin synthesis. Our data indicate that the selected fibronectin-antisense oligonucleotide sequence is specific and effective in reducing fibronectin synthesis in rat endothelial cells.

This study demonstrates the usefulness of fibronectin-antisense oligonucleotides in reversing the effect of high glucose-induced cell proliferation and provides direct evidence that altered fibronectin expression modulates endothelial cell proliferation. Moreover, the findings serve as a basis for future studies on down regulation of gene expression, and underscores the antisense approach as a potential means of investigating the role of basement membrane components in the development of lesions of diabetic microangiopathy.

A cknowledgements. This work was supported by a Career Development Award from the American Diabetes Association.

\section{References}

1. Mosher DF (1984) Physiology of fibronectin. Ann Rev Med 35: 561-75 
2. Roy S, Cagliero E, Lorenzi M (1996) Fibronectin overexpression in retinal microvessels of patients with diabetes. Invest Ophthalmol Vis Sci 37: 258-266

3. Ayo SH, Radnik RA, Garoni JA, Glass WF, Kreisberg JI (1990) High glucose causes an increase in extracellular matrix proteins in cultured mesangial cells. Am J Pathol 136: 1339-1348

4. Madri JA, Pratt BM, Yannarello-Brown J (1988) Matrixdriven cell size change modulates aortic endothelial cell proliferation and sheet migration. Am J Pathol 132: 18-27

5. DiMilla PA, Stone JA, Quinn JA, Albelda SM, Lauffenburger DA (1993) Maximal migration of human smooth muscle cells on fibronectin and type IV collagen occurs at an intermediate attachment strength. J Cell Biol 122: 729 737

6. Cagliero E, Maiello M, Boeri D, Roy S, Lorenzi M (1988) Increased expression of basement membrane components in human endothelial cells cultured in high glucose. J Clin Invest 82: 735-738

7. Roy S, Sala R, Cagliero E, Lorenzi M (1990) Overexpression of fibronectin induced by diabetes or high glucose: a phenomenon with a memory. Proc Natl Acad Sci USA 87: 404-408

8. Roy S, Lorenzi M (1996) Early biosynthetic changes in the diabetic-like retinopathy of galactose-fed rats. Diabetologia 39: 735-738

9. Stein CA, Cheng YC (1993) Antisense oligonucleotides as therapeutic agents - Is the bullet really magical? Science 261: 1004-1012

10. Nagel KM, Holstad SG, Isenberg KE (1993) Oligonucleotide pharmacotherapy: an antigene strategy. Pharmacotherapy 13: $177-188$

11. Vickers T, Baker BF, Cook PD et al. (1991) Inhibition of HIV-LTR gene expression by oligonucleotides targeted to the TAR element. Nucleic Acids Res 19: 3359-3368

12. Becker D, Meier CB, Herlyn M (1989) Proliferation of human malignant melanomas is inhibited by antisense oligodeoxynucleotides targeted against basic fibroblast growth factor. EMBO J 8: 3685-3691
13. Chiang M-Y, Chan H, Zounes MA, Freier SM, Lima WF, Bennett CF (1991) Antisense oligonucleotides inhibit intercellular adhesion molecule 1 expression by two distinct mechanisms. J Biol Chem 266: 18162-18171

14. Schwarzbauer JE, Patel RS, Fonda D, Hynes RO (1987) Multiple sites of alternative splicing of the rat fibronectin gene transcript. EMBO J 6: 2573-2580

15. Felgner PL, Gadek TR, Holm M et al. (1987) Lipofection: a highly efficient, lipid-mediated DNA-transfection procedure. Proc Natl Acad Sci USA 84: 7413-7417

16. Marcum JA, Rosenberg RD (1985) Heparin like molecules with anticoagulant activity are synthesized by cultured endothelial cells. Biochem Biophys Res Comm 126: 365-372

17. Roy S, Roth T (1996) Proliferative effect of high glucose is modulated by antisense oligonucleotides against fibronectin. Diabetes 45 [Suppl 2]:290A (Abstract)

18. Cagliero E, Roth T, Roy S, Lorenzi M (1991) Characteristics and mechanisms of high-glucose-induced overexpression of basement membrane components in cultured human endothelial cells. Diabetes 40: 102-110

19. Roy S, Maiello M, Lorenzi M (1994) Increased expression of basement membrane collagen in human diabetic retinopathy. J Clin Invest 93: 438-442

20. Towbin H, Staehelin T, Gordon J (1979) Electrophoretic transfer of proteins from polyacrylamide gels to nitrocellulose sheets: procedure and some applications. Proc Natl Acad Sci USA 76: 4350-4354

21. Daaka Y, Wickstrom E (1990) Target dependence of antisense oligodeoxynucleotide inhibition of c-Ha-ras p21 expression and focus formation in T24-transformed NIH3T3 cells. Oncogene Res 5: 267-275

22. Zhou L-W, Zhang S-P, Qin Z-H, Weiss B (1994) In vivo administration of an oligodeoxynucleotide antisense to the $\mathrm{D}_{2}$ dopamine receptor messenger RNA inhibits $\mathrm{D}_{2}$ dopamine receptor-mediated behavior and the expression of $D_{2}$ dopamine receptors in mouse striatum. J Pharmacol Exp Ther 268: $1015-1023$ 